\title{
Breaking the Skin Barrier: Current Advancement in Drug Delivery via Skin
}

\author{
Yasser Shahzad ${ }^{1, *}$, Madeeha Malik ${ }^{2, *}$ \\ ${ }^{1}$ North-West University, Centre of Excellence for Pharmaceutical Sciences, Potchefstroom, South Africa \\ ${ }^{2}$ Hamdard University, Hamdard Institute of Pharmaceutical Sciences, Islamabad, Pakistan \\ *Corresponding author: y.shahzad@live.com; madeehamalik15@gmail.com
}

Received August 15, 2014; Revised September 17, 2014; Accepted September 20, 2014

Cite This Article: Yasser Shahzad, and Madeeha Malik, "Breaking the Skin Barrier: Current Advancement in Drug Delivery via Skin." American Journal of Pharmacological Sciences, vol. 2, no. 5A (2014): 0-0. doi: 10.12691/ajps-2-5A.

Human skin provides a protective function by imposing physicochemical limitations to the type of permeant that can navigate the barrier. Transdermal drug delivery offers compelling opportunities to address the low bioavailability of many oral drugs; the pain and inconvenience associated with the injections; and the limited controlled release options of both. For novel delivery methods to succeed and compete with those already on the market, the prime issues that require consideration include device design and safety, efficacy, ease of handling, and cost-effectiveness. Beside this the major challenges and their solutions in designing optimum formulations for skin delivery along with in-depth mechanism relating to the strategies of overcoming the barrier properties of the skin needs to be further explored.

This short special issue of American Journal of Pharmacological Sciences brings together a couple of insightful papers that address some of these issues related to various strategies which have emerged in recent years, to optimise transdermal drug delivery system. The goal of this special issue is to advance the science and methodology in the field of drug delivery and to let the readers become more knowledgeable regarding recent advancements in optimising transdermal drug delivery designs. The first paper has highlighted the importance of ability to incorporate drugs into nanoparticles so as to achieve the ultimate goals of controlled release and sitespecific drug delivery. The authors have emphasised on the need of investigating lipid nanoparticles which might offer possibilities to develop new therapeutic regimens in treatment of complex diseases. The second paper has evaluated the synergistic effect of penetration enhancers, namely propylene glycol (PG) and sodium lauryl Sulphate (SLS) on the transdermal absorption of 2-Methyl-2phenoxy-propionic acid (MPA) with the aid of a statistical method, namely response surface methodology (RSM). The paper reported the combination of PG and SLS to be utilised successfully as permeation enhancers for transdermal drug delivery of MPA with minimal side effects. 